\title{
What every doctor wants to know about cholesterol: answers to some difficult questions
}

\author{
Edited by P.N. Durrington
}

\section{Introduction}

\section{P.N. Durrington}

University of Manchester Department of Medicine, Manchester Royal Infirmary, Oxford Road, Manchester M13 9WL, UK

I recently had the privilege of asking leading authorities in Britain for their views on some of the difficult questions that regularly arise in the context of the clinical management of hyperlipidaemia. These were to be brief, yet contain the key points which might be used in responding to questions at medical meetings. The whole idea arose at a committee meeting of the British Hyperlipidaemia Association (BHA), and Professors J. Shepherd of Glasgow University and A.F. Winder from the Royal Free Hospital joined me in formulating the list of topics and the experts whom I approached. Members of the committee of the BHA have subsequently commented on some of the answers as part of the editorial process.

The treatment of hypercholesterolaemia has recently received major attention not only in the medical press, but also in national newspapers, as a result of publicity attending two meta-analyses of lipid-lowering drug trials in primary coronary prevention. ${ }^{1,2}$ Much of the public debate has focused on the negative aspects of these analyses, when in reality they complement the evidence established in individual trials that reducing cholesterol decreases the end point of coronary morbidity by also establishing that it decreases coronary mortality. Furthermore they establish that lipid-lowering therapy does not increase the risk of cancer death. There is the suggestion from the meta-analyses that lipid lowering may have increased non-medical deaths: suicides, accidents and homicides. It is this aspect of the reports which has received most publicity. It should be pointed out that one study which lasted only one year was included $^{3}$ and that this contributed almost one third of the non-medical deaths from all the trials included in the analyses. Two other studies of only one year ${ }^{4}$ and 2 years ${ }^{5}$ were also included and even the most enthusiastic supporter of the cholesterol hypothesis (and the authors of these meta-analyses were certainly not that) would not have expected any benefits in such a short time. Furthermore can we really accept that non-medical deaths can be considered together? Have they really as much in common in their causation as deaths due to coronary heart disease (CHD) or to malignancy might? What single hypothesis could possibly explain the deaths of one man who is the passenger in an air crash and another who commits suicide? In this series of answers, Professor Shaper takes a close look at the association between spontaneously low cholesterol levels and non-CHD deaths and finds little to suggest a causal link. Dr Keech provides an early insight into a more comprehensive meta-analysis than any so far published involving more than 20 trials and indicating that some of the more controversial findings of the earlier analyses were due to random effects when there are relatively small numbers of end-points in individual trials. Dr Thompson discusses the possibility that some members of one group of drugs used to lower cholesterol, the statins, might affect central nervous system function.

All of the trials of cholesterol-lowering in primary CHD prevention were conducted in men, many of whom had much lower cholesterol levels and coronary risk than would be an indication for lipid-lowering drugs, if in clinical practice recommendations of bodies such as the British Hyperlipidaemia Association were followed. ${ }^{6}$ Thus in the WHO trial $^{7}$ the mean cholesterol at randomization was only $6.4 \mathrm{mmol} / 1$, in the LRC study it was $7.2 \mathrm{mmol} / 1^{8}$ and in the Helsinki Heart Study ${ }^{9}$ it was $6.9 \mathrm{mmol} / 1$. Reductions of only $8.5-10 \%$ in cholesterol were achieved, despite which the decrease in coronary mortality and morbidity ranged from 19 to $34 \% .^{7-9}$ The trials were analysed on an intention-to-treat basis which is quite correct if one is testing the scientific hypothesis that cholesterol reduction leads to a decrease in coronary morbidity which they were designed to do, but is certainly not what we do in clinical practice. If a patient has an inadequate decrease in serum cholesterol with one medication we do not continue it indefinitely. We choose a drug which is more acceptable and effective in that individual. This means that estimates of cost-effectiveness based on a literal interpretation of the results of the trials will be a gross 
overestimate of cost. This point is clearly made by Dr Reckless in his article in this series. If, as clinicians, we choose to treat people with higher cholesterol levels or with additional risk factors who are thus at greater CHD risk and to use effective cholesterol-lowering medication then there is every reason to believe that a much greater reduction in the risk of both fatal and non-fatal CHD than occurred in the trials will be achieved in practice. On the other hand any adverse effects, because they are unrelated to cholesterol reduction, will remain at a similar low level to those in the trials. The balance of benefit and risk is thus likely to favour cholesterol-lowering intervention. ${ }^{10} \mathrm{Dr}$ Durrington discusses this approach in one special high-risk group of patients: those who have already developed CHD. It is essential that patients with cholesterol levels of only $6.5 \mathrm{mmol} / \mathrm{l}$ are not given lipid-lowering medication when this is their only risk factor. This is clearly highlighted by the meta-analyses. When an elevated serum cholesterol is the only risk factor for CHD, a level greater than $8.0 \mathrm{mmol} / 1$ despite diet is probably when lipidlowering drugs should be considered. The CHD risk in such an individual would be similar to one with a cholesterol of $6.5 \mathrm{mmol} / 1$ combined with hypertension and a history of smoking, or another with diabetes or with a family history of CHD.

When and in whom to screen for hyperlipidaemia remains a subject of much debate and the issues raised at either end of the age scale and by women with lower CHD risk than men are dealt with by Drs West and Miller. Women also pose special problems in the management of hyperlipidaemia with regard to oral contraception and the appropriateness of hormone replacement therapy. Dr Seed discusses these questions. Dr Betteridge discusses whether to use thiazide diuretics or beta blockers in the management of hypertension when other agents having less potentially adverse effects on serum lipoproteins and Dr Sanders tackles the perennial question of whether fish oil has a part to play in CHD prevention.

Oliver said in 1978 'What is clear, at least, is that reduction of serum cholesterol concentration is relevant to the prevention of coronary heart disease."11

More recently, Steinberg commented that 'Hypercholesterolaemia, while certainly not the only cause of coronary heart disease, is equally certainly one of its major correctable causes... There is still room for discussion of the cholesterol level at which treatment is called for and of just how vigorously it should be pursued. ${ }^{12}$ This is where the debate in Britain should now centre. For those engaged in the practice of CHD prevention, this series of answers to difficult questions should prove helpful.

\section{References}

1. Muldoon, M.F., Manuck, S.B. \& Matthews, K.A. Lowering cholesterol concentrations and mortality: a quantatitve $C$ review of primary prevention trials. $\mathrm{Br} \mathrm{Med} J$ 1990, 301: 309-314.

2. Smith, G.D. \& Pekkanen, J. Should there be a moratorium on the use of cholesterol lowering drugs? Br Med J 1992, 304: $431-434$.

3. Frantz, I.D., Dawson, E.A., Achman, P.L. et al. Test of effect of lipid lowering by diet on cardiovascular risk. Arteriosclerosis 1989, 9: 129-135.

4. Dorr, A.E., Gundersen, K., Schneider, J.C., Spencer, T.W. \& $\mathrm{\&}^{\text {బ) }}$ Martin, W.B. Colestipol hydrochloride in hypercholestero- $\overrightarrow{0}$ laemic patients - effect on serum cholesterol and mortality. $J_{-}$ Chronic Dis 1978, 31: 5-14.

5. Bradford, R.H., Shear, C.L., Chremios, A.N. et al. Expanded clinical evaluation of lovastatin (EXCEL) study results. I.D Efficacy in modifying plasma lipoproteins and adverse event profile in 8,245 patients with moderate hypercholesterol․․‥ aemia. Arch Intern Med 1991, 151: 43-49.

6. Shepherd, J., Betteridge, D.J., Durrington, P.N. et al. $\infty$ Strategies for reducing coronary heart disease and desirable limits for blood lipid concentrations: guidelines of the British. Hyperlipidaemia Association. $\mathrm{Br} \mathrm{Med} J$ 1987, 295: 1245-ळ
1246.

7. Committee of Principal Investigation. A cooperative trial in음 the primary prevention of ischaemic heart disease using clofibrate. Br Heart $J$ 1978, 40: 1069-1118.

8. Lipid Research Clinics Program. The Lipid Research Clinics coronary primary prevention trial results. JAMA 1984, 251:\$ $351-374$.

9. Frick, M.H., Elo, O., Haapa, K. et al. Helsinki Heart Study: primary prevention trial with gemfibrozil in middle-aged men with dyslipidaemia. $N$ Engl J Med 1987, 317: 1237-124ㅇㅇㅇ $\overrightarrow{0}$

10. Durrington, P.N. Hyperlipidaemia: should we treat patienזs? Should we treat populations? What treatment should we usis? ? In: Rowlands, D.J. (ed.) Recent Advanced in Cardiology, $\bar{B} \mid \square$ II. Churchill Livingstone, Edinburgh, 1992, pp. 47-71.

11. Oliver, M.F. Cholesterol, coronaries, clofibrate and death. $N \leqq$ Engl J Med 1978, 299: 1361.

12. Steinberg, D. Foreword. In: Durrington, P.N. (ed.) Hyperlipidaemia Diagnosis and Management. Wright, London, $\stackrel{\mathbb{D}}{\mathrm{Q}}$ 1989, p. v.

Low cholesterol and non-cardiac death; cause or effect?

\section{A.G. Shaper}

Department of Public Health and Primary Care, Royal Free Hospital School of Medicine, Rowland Hill Street, London NW3 2PF, UK

In every country in which the average blood cholesterol is relatively high (greater than $5-6$ 을. $\mathrm{mmol} / \mathrm{l}$ ) there is a strong, direct association $\bar{N}$ between blood cholesterol level in individuals and $\%$ coronary heart disease (CHD). A large prospective ${ }^{N}$ study of Chinese men and women aged 35-64 N years at baseline and followed for 8-13 years, has 0 now examined the blood cholesterol relationship inco a population with a much lower average level than $\frac{}{\Phi}$ in Western populations, viz. $4.2 \mathrm{mmol} / 1 .{ }^{1}$ There $\stackrel{?}{?}$ was a strong, positive and independent relation 0 between blood cholesterol and CHD deaths, with no evidence of any threshold of risk within the 
range encountered, viz. $3.8-4.7 \mathrm{mmol} / 1$. There was no association between blood cholesterol concentration and death from stroke, even among subjects with a raised blood pressure. There was also no significant association between baseline blood cholesterol and total cancer mortality, but there was an inverse trend for liver cancer with the highest risk ( $60 \%$ increase) observed in the lowest quarter of cholesterol concentration. The same inverse pattern of association was present for chronic liver disease. The association between these two conditions and low blood cholesterol concentration probably reflects the long duration of the key factor underlying the development of both these conditions, persistent hepatitis B infection. There was a marginally significant inverse association between death attributed to non-medical causes (accidents, injury, suicide) and blood cholesterol concentration. The number of these deaths was small, no single cause was predominant and the association was non-significant after other variables were taken into account. In the Renfrew and Paisley survey in Scotland, an inverse association between blood cholesterol and cancer in men was demonstrated, strongest for lung cancer. ${ }^{2}$ The authors concluded that dietary advice given to the population to reduce the intake of saturated fat might increase overall mortality.

In order to assess the evidence for a long-term association of low blood cholesterol with cancer, Law and Thompson have analysed data from 33 prospective studies. ${ }^{3}$ For short-term cancers in men (diagnosis within 2 years or death within 5 years of cholesterol measurement) there was a $70 \%$ increase in risk in the lowest cholesterol quintile. For long-term cancers there was only a $15 \%$ increase in risk, although this was highly significant. There was considerable variation between the results in the different studies, and the long-term association was pronounced only in studies of manual workers. In women, there was a $10 \%$ increase in the long term risk of cancer in the lowest cholesterol quintile, but no social class effect was seen. Lung cancer appeared to account for the long-term association of low cholesterol with all cancers in men, but not women. For all other cancers, the associations were not significant for men or women.

The authors focus on cigarette smoking as the possible explanation for the association of lung cancer with low blood cholesterol. Smoking certainly lowers high density lipoprotein (HDL)cholesterol concentrations, but total cholesterol levels tend to be similar in smokers and nonsmokers. They conclude that the long-term inverse association is small and very much smaller than the direct association with CHD. Furthermore, there is no direct evidence that the association with cancer is causal and 'dietary recommendations to lower serum cholesterol should be given unreservedly'.
The British Regional Heart Study data may throw some light on the association between low blood cholesterol and cancer, and low blood cholesterol and non-medical conditions (accident, injury, suicide). As assessment was made of the relationship between alcohol intake, cigarette smoking, body weight and blood lipid concentrations in 7,735 men aged 40-59 years. ${ }^{4}$ With the exception of HDL-cholesterol, the relationship between alcohol intake and blood lipids (cholesterol, triglyceride) was significantly different in smokers and non-smokers, apparently due to the opposing effects of smoking on blood lipids and body weight. The lowest blood cholesterol levels were seen in current smokers who were heavy drinkers ( $>42$ drinks/week) or non-drinkers, the latter group including a high proportion of exdrinkers and men with a high prevalence of illhealth. The reported association between low blood cholesterol levels and increased mortality or increased prevalence of non-medical conditions may reflect the burden of disease and disturbance in heavy drinkers, who are cigarette smokers, and in ex-drinkers who are current smokers. This could explain the social class effect observed by Law and Thompson. In the paper by Muldoon et al. (1990) attention is drawn to low serum cholesterol levels 'among criminals, people with diagnoses of violent or aggressive conduct disorders, homicidal offenders with histories of violence and suicide attempts related to alcohol, and people with poorly internalized social norms and low self control."5 Alcohol, cigarette smoking and social-economic status might go a long way to explain the associations between all these outcomes and low blood cholesterol concentrations.

\section{References}

1. Chen, Z., Peto, R., Collins, R., MacMahon, S., Lu, J. \& Li, W. Serum cholesterol concentration and coronary heart disease in a population with a low cholesterol concentration. $\mathrm{Br} \mathrm{Med} \mathrm{J}$ 1991, 303: 276-282.

2. Isles, C.G., Hole, D.J., Gillis, C.R., Hawthorne, V.M. \& Lever, A.F. Plasma cholesterol, coronary heart disease, and cancer in the Renfrew and Paisley survey. Br Med J 1989, 298: 920-924.

3. Law, M.R. \& Thompson, S.G. Low serum cholesterol and the risk of cancer: an analysis of the published prospective studies. Cancer Causes Control 1991, 2: 253-261.

4. Wannamathee, G. \& Shaper, A.G. Blood lipids: the relationship with alcohol intake, smoking and body weight. $J$ Epidemiol Commun Health 1991, 46: 197-202.

5. Muldoon, M.F., Manuck, S.B. \& Matthews, K.A. Lowering cholesterol concentrations and mortality: a quantitative review of primary prevention trials. $\mathrm{Br}$ Med J 1990, 301: 309-314. 


\section{Does cholesterol lowering reduce total mortality?}

\section{A. Keech \\ Oxford Cholesterol Study, The Harkness Building, Radcliffe Infirmary, Oxford $O X 26 \mathrm{HE}, U K$}

Even collectively, the existing trials of cholesterol lowering have, perhaps surprisingly, not yet tested reliably for the net effects on overall survival that would be reasonably expected from available evidence. It is chiefly low statistical power to examine total mortality, notwithstanding the small nonsignificant excesses of various non-coronary heart disease (CHD) causes of death in the trials to date, that leaves the issue unresolved.

\section{A lack of power}

More than 20 clinical end-point trials studying the effects of cholesterol lowering have been conducted over the last few decades and they leave no doubt that the risk of CHD is rapidly reduced. The most reliable estimates come from meta-analyses or overviews, reducing random error and increasing power by combining the results of all related trials, provided that publication (and other) biases are avoided. In secondary prevention, a $12 \%(2 P<$ 0.05 ) reduction in odds of fatal CHD has been reported, ${ }^{1}$ and in an overview of some of the primary prevention trials (limited to males) a similar $15 \%(P=0.06)$ reduction was seen. ${ }^{2}$ The most comprehensive overview to date, of 22 published and unpublished randomized studies involving a total of about 42,000 subjects $^{3}$ (R. Peto, personal communication, 1991) shows a highly significant $(16 \% \pm 3 ; 2 P<0.0001)$ reduction in CHD events from a $10 \%$ lowering of cholesterol, achieved for an average of only 1-3 years in those suffering a CHD event in the trials. In contrast, only a small non-significant $3 \%$ reduction in total mortality was seen. However, this is not proof that there is no effect on overall survival, because the confidence intervals of the estimate (from an $8 \%$ reduction to a $3 \%$ increase) are compatible with the modest $6 \%$ reduction in total mortality expected to result from the observed reduction in CHD mortality (since many people died of conditions other than CHD).

To date, the cholesterol differences achieved in the trials have been small (average around $10 \%$ ), and for only a very short duration; hence, the reductions in CHD mortality have only been small. Additionally, the number of deaths has been small, with very low rates of fatal CHD in the primary prevention trials, where many more non-CHD deaths may dilute or abolish the statistical significance of the effect of reduced CHD mortality on total mortality. For example, consider the overview? of some of the primary prevention trials by $\mathrm{Mul}-\overline{\mathrm{D}}$ doon et al., ${ }^{2}$ in which the cholesterol reduction ${ }_{C}$ averaged $10 \%$ and the risk of death was low, with CHD death rates outnumbered $2: 1$ by non-CHDon? rates; approximately $1.5 \%$ and $3.1 \%$, respectively, over 4.8 years. To have had a good probabilityo (that is, $90 \%$ 'power') of detecting a conventionally $\frac{}{5}$. significant $(2 P<0.05)$ reduction in total mortality from the $15 \%$ reduction in odds of fatal CHD seeno (assuming there are no materiai effects on non- CHD death) would have required more than: 300,000 participants, as opposed to the $25,000-\overrightarrow{\mathrm{\omega}}$ reviewed (with only $14 \%$ power). Similarly, in theo POSCH study ${ }^{4}$ where a much larger $23 \%$ choles? terol reduction was achieved through ileal bypass surgery, a $21 \%(P=0.16)$ reduction in total mor-क tality resulted from a $27 \%$ fall in fatal CHD, but to have had a good $(90 \%)$ probability of achievingor conventional significance in the trial for such ao result would have needed not 838 but 4,800 parti- $v$ cipants. Thus, even when considered together in음 overviews, such study results clearly lack the necessary power to discern reliably whether reduc- $z$ ing CHD can reduce total mortality.

\section{The safety of cholesterol lowering}

Muldoon et al., ${ }^{2}$ Oliver $^{5}$ and Davey Smith et (who included non-randomized results) have raisedo the question of whether the clear benefit of lipids lowering with regard to coronary heart disease iso offset by adverse effects on other conditions so thato total mortality is not reduced. Frequently cited $\mathbb{B}$ concerns are the excess of cancer deaths reported in $\overrightarrow{\vec{B}}$ the WHO clofibrate study (not using standardB 'intention-to-treat' analysis), ${ }^{7}$ and of violent/accidental deaths in the Lipid Research Clinic study ${ }^{8}$ of cholestyramine, or in the Helsinki Heart Study of gemfibrozil. ${ }^{9}$ Yet in the comprehensive meta-ana 3 . lysis of unconfounded trials by Peto et al., which includes all of the latter studies (and uses 'inten tion-to-treat' analyses), a marginally significantô excess of non-CHD deaths overall is seen, but no cause of non-CHD death (for example, cancer, or? violent, suicide and non-illness-related deaths) $>$ taken separately is individually significant or im을. pressive. Hence, although it cannot be disproved that the excesses of non-CHD death might reflecto real hazard, good evidence upon which a conclus $N$ ion of real hazard should be based is lacking when $N_{\omega}$ all the available data are examined, and it remains perfectly plausible that these excesses are due to theo play of chance alone. The data in the trials to date do not allow us to distinguish with certainty? between these who possibilities. So we must awaito larger trials with big reductions in cholesterol and more end-points to settle this issue. Other evidences 
is reassuring; a five-year follow-up of the Merck Sharp and Dohme experience with larger cholesterol reductions (up to $30 \%$ ) using lovastatin shows no excess of death from violence or cancer. ${ }^{10}$ The (so far) largest and longest reduction in cholesterol (23\% and 10 years) has been achieved in the POSCH trial, ${ }^{4}$ and this shows no excess cancer or violent deaths, although the numbers of such events have been small.

The evidence for clinically important reduction in CHD risk by lowering cholesterol is overwhelmingly statistically significant (five standard deviations from zero effect). But, until large cholesterol differences (for example, such as those now conveniently available with the HMG CoA reductase inhibitors ${ }^{11,12}$ ) potentially capable of causing much bigger reductions in coronary mortality can be tested long-term in studies, which will yield many more CHD and non-CHD deaths than seen in previous trials, we are not likely to have adequate power to resolve the uncertainty about the effects of cholesterol lowering on total mortality, and about its safety, once and for all. It seems prudent therefore to restrict the use of lipid-lowering medication at the present time to patients who are at higher risk of CHD death than those in the primary prevention trials and whose chances of dying prematurely of CHD would otherwise be more likely than their chance of dying from some other cause.

\section{References}

1. Rossouw, J.E., Lewis, B. \& Rifkind, B.M. The value of lowering cholesterol after myocardial infarction. $N$ Engl $J$ Med 1990, 323: 1112-1119.

2. Muldoon, M.F., Manuck, S.B. \& Matthews, K.A. Lowering cholesterol concentrations and mortality: a quantitative review of primary prevention trials. $\mathrm{Br}$ Med $J$ 1990, 301: 309-314.

3. Peto, R., Yusuf, S. \& Collins, R. Cholesterol-lowering trial results in their epidemiologic context. Circulation 1985, 72: 451.

4. Buchwald, H., Vargo, R.L., Matts, J.P. et al. \& the POSCH Group. Effect of partial ileal bypass surgery on mortality and morbidity from coronary heart disease in patients with hypercholesterolemia. Report of the Program on the Surgical Control of the Hyperlipidemias (POSCH). N Engl J Med 1990, 323: 946-955.

5. Oliver, M.F. Might treatment of hypercholesterolaemia increase non-cardiac mortality? Lancet 1991, 337: 15291531.

6. Davey Smith, G. \& Pekkanen, J. For debate: Should there be a moratorium on the use of cholesterol lowering drugs? $\mathrm{Br}$ Med J 1992, 304: 431-443.

7. Report from the Committee of Principal Investigators. A cooperative trial in the primary prevention of ischaemic heart disease using clofibrate. Br Heart J 1978, 40: 1069-1118.

8. Lipid Research Clinics Coronary Prevention Trial. I. Reduction in incidence of coronary heart disease. II. The relationship of reduction in incidence of coronary heart disease to cholesterol lowering. JAMA 1984, 251: 351-374.

9. Frick, M.H., Elo, O., Haapa, K. et al. Helsinki Heart Study: Primary-prevention trial with gemfibrozil in middle-aged men with dyslipidemia. N Engl J Med 1987, 317: 1237-1245.
10. Tobert, J.A. Cholesterol lowering and non-cardiac mortality (Letter). Lancet 1991, 338: 126.

11. Keech, A. (for the Oxford Cholesterol Study Group). Randomised trial of the effects of cholesterol lowering with simvastatin in patients at increased risk of coronary heart disease. Atherosclerosis 1990, 85: 92.

12. Bradford, R.H., Shear, C.L., Chemros, A.N. et al. Expanded clinical evaluation of lovastatin (EXCEL) study results. Arch Intern Med 1991, 151: 43-49.

\section{Statins and the central nervous system}

\section{G.R. Thompson}

\section{MRC Lipoprotein Team, Hammersmith Hospital, London, UK}

Cholesterol is an important constituent of the central nervous system (CNS) and comprises about $20 \%$ of the non-exchangeable pool of cholesterol in the body. Most of the cholesterol in the CNS is derived from local synthesis, rates being high in the developing fetus and during early infancy but very low in adult brain. The latter's cholesterol content does not appear to be influenced by changes in plasma cholesterol brought about by diet or nonabsorbable cholesterol-lowering drugs such as cholestyramine. However, this does not mean that the CNS in adults is metabolically inert in respect of lipids. Although low density lipoprotein (LDL) is too large to cross the blood-brain barrier, small sized high density lipoprotein (HDL) particles have been identified in the cerebrospinal fluid. Furthermore, Mahley ${ }^{1}$ has described in detail the important role played by apolipoprotein $\mathrm{E}$ in repairing damaged nerves. Thus it is pertinent to consider whether drugs which decrease cholesterol synthesis by inhibiting HMG CoA reductase, collectively referred to here as statins, might adversely affect CNS function.

The two statins licensed for use in Britain are simvastatin (Zocor) and pravastatin (Lipostat). Simvastatin is a close analogue of lovastatin (Mevacor), both drugs being administered as inactive lactones which subsequently get converted to open acids, whereas pravastatin is administered as the biologically active acid. Other differences are that lovastatin and simvastatin and their metabolites are more lipophilic than pravastatin, whereas the latter is less completely protein-bound in plasma. Despite these differences the cholesterol-lowering ability and side effect profile of the two classes of statin are remarkably similar in most respects. ${ }^{2}$ There is controversy, however, on the possible influence of these drugs on CNS function, which stems from a claim that lovastatin disturbed sleep whereas pravastatin did not. ${ }^{3}$ This was attributed to the ability of the former drug to cross the 
blood-brain barrier. Subsequent studies of small numbers of subjects have given equivocal results, ${ }^{4,5}$ although the large EXCEL study showed no difference in sleep patterns between lovastatin and placebo under double-blind conditions. ${ }^{6}$ However, two recent studies suggested that lovastatin adversely affected daytime performance whereas prevastatin did not but again the numbers involved were relatively small. ${ }^{7.8}$

What does seem beyond contention is that lovastatin penetrates the blood-brain barrier to a greater extent than pravastatin. ${ }^{9}$ This was demonstrated by subjecting 12 normolipidaemic volunteers to lumbar punctures at intervals after single doses of either drug, and assaying the concentration and inhibitory activity of each drug and its metabolites in the serum and in cerebrospinal fluid. Amounts of lovastatin corresponding to $13-18 \%$ of its concentration in serum were found in the cerebrospinal fluid from four of six subjects on GC-mass spectrometry but no inhibitory activity against HMG CoA reductase was detectable on bioassay. In contrast, trace amounts of a pravastatin metabolite were found in only one of six subjects, again without any inhibitory activity on bioassay.

At present it is difficult to know whether these differences are academic or whether the ability of lipid-soluble statins like lovastatin, and presumably simvastatin, to penetrate the blood-brain barrier could result in CNS dysfunction. The answer to this question is far from academic in view of the suggestion that cholesterol-lowering therapy may induce changes in the lipid composition of cell membranes which might result in an enhanced predisposition to accidental death. ${ }^{10}$ The evidence so far provides no support for this hypothesis with respect to lovastatin, ${ }^{11}$ the statin with which the greatest experience has been obtained to date, but long-term observations are necessary before it can be totally refuted.

\section{References}

1. Mahley, R.W. Apolipoprotein E: cholesterol transport protein with expanding role in cell biology. Science 1988, 24: 622-630.

2. Tobert, J.A. The HMG-CoA reductase inhibitors. Similarities and differences. In: Tarr, I. (ed.) Anti-atherosclerotic Agents Current Drugs, 1991, pp. B21-B29.

3. Schaefer, E.J. HMG-CoA reductase inhibitors for hypercholesterolemia (letter). $N$ Engl J Med 1988, 319: 1222.

4. Kales, A., Bixler, E.O., Manfredi, R.L. et al. Effects of lipophilic vs nonlipophilic drugs on nighttime sleep (abstract). World Congress Psychiatric Association Meeting, Athens, October 1989, Abstract No. 2676.

5. Eckernas, S.-A., Roog, B.-E., Kvidal, P. et al. Clinical and sleep EEG study of two structurally different HMG CoA reductase inhibitors (abstract). Proceedings of the 9 th International Symposium on Atherosclerosis, Chicago, October 1991, p. 113.
6. Bradford, R.H., Shear, C.L., Chremos, A.N. et al. Expanded clinical evaluation of lovastatin (EXCEL) study results. Efficacy in modifying plasma lipoproteins and adverse everf profile in 8,245 patients with moderate hypercholesterolemia.. Arch Intern Med 1991, 151: 43-49.

7. Richardson, G., Sullivan, J. \& Lee, R. Changes in daytimê performance following lovastatin, pravastatin, and placebo administration (abstract). Proceedings of the 9 th Internationat Symposium on Atherosclerosis, Chicago, October $199 \mathrm{E}$. p. 116.

8. Roth, T., Merlotti, L. \& Roehrs, T. Comparative effects $\$$ lovastatin and pravastatin administration on daytime perfor mance (abstract). Proceedings of the 9th International Sym posium on Atherosclerosis, Chicago, October 1991, p. 116. $\overrightarrow{0}$

9. Botti, R.E., Triscari, J., Pan, H.Y.\& Zayat, J. Concentrations. of pravastatin and lovastatin in cerebrospinal fluid in health
subjects. Clin Neuropharm 1991, 14: 256-261.

10. Oliver, $M$. Might treatment of hypercholesterolaemia iñ̄ crease non-cardiac mortality? Lancet 1991, 337: 1529-153\%

11. Tobert, J.A. Cholesterol-lowering and non-cardiac mortality. (letter). Lancet 1991, 338: 126.

Treatment of hyperlipidaemia in patients with pre-existing coronary heart disease

Paul N. Durrington

University of Manchester, Department of Medicine $\frac{\mathbb{1}}{\mathrm{S}}$ Oxford Road, Manchester M13 9WL, UK

\section{Evidence of benefit $^{1}$}

The two most important primary prevention trial of cholesterol lowering, the Lipid Research Clinio (LRC) Study and the Helsinki Heart Study, estabe lished clearly that coronary heart disease (CHD\$ morbidity can be reduced by this means. The middle-aged men who participated in these trials were, however, at lower risk of CHD than middle? aged men in general. This was largely because those with electrocardiographic evidence or a history of CHD were excluded and we know from the Britisis Regional Heart Study that such men comprise large proportion of those destined to die fromp CHD in the next 5 years. It is notable that in botk trials there was a progressively widening difference in the combined end-point of fatal and non-fatat myocardial infarction between men on placebo ante. active therapy throughout. In other words the benefits of lipid-lowering therapy became more obvious as the effects of flushing out men with pre-existing CHD from the study populations wores off and previous occult coronary disease pro gressed. The trials indicate that if we want too influence overall mortality as well as CHD morbid ity with lipid-lowering therapy we need to turn to people at higher risk than the study populations of the LRC and Helsinki Heart Study. Such people might be those with higher cholesterol levels (the? 
average cholesterol when lipid-lowering therapy was administered was only $6.9 \mathrm{mmol} / 1$ in the Helsinki and $7.2 \mathrm{mmol} / \mathrm{l}$ in the LRC trial) or with other risk factors combined with hypercholesterolaemia such as a bad family history of CHD, diabetes, hypertension, long smoking history and in particular a personal history or evidence of pre-existing CHD. The wisdom of the latter was well tested in the Stockholm Secondary Prevention Trial.

In the Stockholm trial 555 patients $(20 \%$ women), who had survived myocardial infarction, were randomly assigned to receive lipid-lowering medication (clofibrate and nicotinic acid) or placebo. There was a $13 \%$ decrease in serum cholesterol and a $19 \%$ reduction in serum triglyceride levels. In this high-risk group a $26 \%$ decline in all-cause mortality stemming from a $36 \%$ fall in CHD mortality was evident over the 5 years of the study. A meta-analysis of all the secondary prevention trials indicated that this result closely matches the overall outcome of such trials. ${ }^{2}$ Thus, if we choose to treat patients whose likelihood of dying from CHD is substantially greater than from any other cause, we can influence all-cause mortality.

Some studies of cholesterol-lowering therapy which may provide additional mortality data in patients with pre-existing CHD are in progress and have yet to report. However, clinicians who stand back in the hope of having a significant 'dead-body count' at the end of such trials are evading their responsibility for making up their minds largely on the basis of existing evidence. This is because patients with CHD or who develop its complications in current trials cannot reasonably be denied the benefits of new cardiovascular drugs and of myocardial revascularization by surgery or angioplasty. This means that CHD mortality in any closely scrutinized group of patients will be kept low. This is well illustrated by the recent results from the Program on the Surgical Control of the Hyperlipidaemias (POSCH). Like the earlier Stockholm study this was conducted in myocardial infarction survivors, but, whereas the deaths in the Stockholm Study were $26 \%$ over 5 years, in POSCH only $13 \%$ died in twice as long. Patients with left mainstem disease were excluded at the outset. Then during the course of the trial more than a fifth of patients had coronary artery bypass grafting on clinical grounds and other medical advances in the treatment of CHD since the earlier Helsinki trial were not withheld. Thus, although there were fewer deaths in the group treated for hypercholesterolaemia, the total deaths were too few for statistical significance.

Increasingly we shall have to take into account the results of coronary angiographic studies. Three early studies using cholestyramine or clofibrate and nicotinic acid or diet all showed decreased progres- sion of coronary disease. The two most recent ones employing the combination of niacin and colestipol, or colestipol and lovastatin showed in addition evidence of regression.

It is noteworthy that in the Stockholm trial and the coronary angiographic studies, benefit appeared to occur at lower levels of cholesterol than the $6.5 \mathrm{mmol} / \mathrm{l}$ which is the currently recommended lower level for consideration of drug therapy and also there may have been benefit from lowering triglycerides as well as cholesterol. It may be therefore that we should be prepared to consider lipid-lowering therapy at lower levels in patients with pre-existing CHD and that our therapeutic targets should also be lower - say less than $5 \mathrm{mmol} / \mathrm{l}$ for cholesterol.

\section{Coronary artery surgery}

Patients who have undergone coronary artery surgery are an important group since several investigations have now shown that hyperlipidaemia is the dominant factor in graft occlusion and progress of disease in the native coronary circulation. It seems reasonable that all such patients should be carefully screened for hyperlipidaemia and that when this is present they should be treated regardless of age. (If they are fit enough to undergo coronary surgery they must be biologically young enough for lipid-lowering therapy to extend their lives.)

\section{Coronary angioplasty}

The atheroma which reoccludes coronary vessels following angioplasty is of the 'white' (fibrous) type, which does not contain cholesterol. There is no evidence that lipid-lowering therapy will prevent this complication. However, the angiographic studies clearly demonstrate that lipid-lowering therapy will impede or regress atheroma in other parts of the coronary circulation and for this reason it should always be considered in patients undergoing coronary angioplasty.

\section{Poor cardiovascular prognosis}

Obviously patients whose CHD is associated with severe heart failure requiring large doses of diuretic and/or angiotensin converting enzyme inhibitors do not have a sufficiently long life expectancy to benefit from lipid-lowering drugs. Dietary restrictions and unnecessary medication may well impair the quality of their limited life and should be avoided. 


\section{When to measure lipids ${ }^{3}$}

There is evidence that the fall in serum cholesterol and the rise in triglyceride that accompany acute myocardial infarction, largely occur after the first 24 hours. It has therefore been advocated that lipid levels should be measured on admission to the coronary care unit. Often in practice it is not easy to be sure that the blood has been taken within the first day or to time the exact onset of the myocardial infarction. It is essential therefore to check lipids some 2 months after myocardial infarction. Even then, however, they may not have returned to their pre-infarction level, possibly because of angina. It is not uncommon to discover hyperlipidaemia for the first time after recovery from successful coronary artery bypass surgery, and all patients undergoing this operation should have their serum lipids checked 3 months afterwards regardless of any earlier normal results.

\section{Aspirin $^{4}$}

This should not be omitted from the treatment regimen of patients attending the Lipid Clinic with pre-existing CHD.

\section{Diet}

Whilst there is little clinical trial evidence for the benefit of a lipid-lowering diet (as opposed to drugs) after myocardial infarction, it is nonetheless important: firstly because obesity increases cardiac work, and secondly because lipid-lowering drug therapy is usually only fully effective when combined with dietary therapy.

\section{References}

1. Durrington, P.N. Hyperlipidaemia: should we treat patients? Should we treat populations? What treatment should we use? In: Rowlands, D.J. (ed.) Recent Advances in Cardiology, Vol. 11. Churchill Livingstone, Edinburgh, 1992, pp. 47-71.

2. Rossouw, J.E., Lewis, B. \& Rifkind, B.M. The value of lowering cholesterol after myocardial infarction. $N$ Engl $J$ Med 1990, 323: 1112-1119.

3. Durrington, P.N. Normal serum lipid and lipoprotein concentrations. In: Hyperlipidaemia. Diagnosis and Management. Wright, London, 1989, pp. 56-86.

4. Waters, D. \& Lesperance, J. Regression of coronary atherosclerosis: an achievable goal? Review of results from recent clinical trials. Am J Med 1991, 91 (Suppl 1B): 105-175. Screening for hyperlipidaemia and its treatment in
childhood

\section{R. West}

Medical Postgraduate Department, Canynge Hall, Whiteladies Road, Bristol BS8 2PR, UK

With any screening programme it is necessary t establish that the disease is an important health problem, can be recognized in a presymptomatic of early stage, that facilities exist for diagnosis and treatment with sufficient resources earmarked, and treatment must be known to confer benefit. Wheip considering lipid screening in childhood with the aim of preventing adult coronary heart disease. (CHD) there are two possible approaches; screen: ing can be targeted towards individuals an $\delta_{0}$ families suspected of being at particular risk, of might be population based. These will be conio sidered separately.

\section{Targeted screening}

Early onset CHD is common in inherited choles terol or other lipid problems, and has a significan mortality. ${ }^{1}$ Lipid studies should be done in all patients with early onset CHD; these will identy whether or not there is a lipid abnormality. Fan studies will help in determining whether the prob응 lem is familial. Children should be screened as parE of any such family studied. Targeted screening fo $\bar{D}$ lipid abnormalities is also indicated in first of̆ second degree relatives where the index patient is known to have had early onset coronary hear disease, even if they are not available for lipis testing, and also where another close family member is known to have hyperlipidaemia.

The purpose of targeted screening in childhood is to detect whether a child has an inherited lipid. problem predisposing to premature CHD. Familiab hypercholesterolaemia (FH) and familial com? bined hyperlipidaemia (FCH) are the main condio tions; both are relatively common in the families of patients having myocardial infarction at an early? age. The risk of developing CHD in early adult life in patients with $\mathrm{FH}$ is much greater than the risk in. acquired hyperlipidaemia. Targeted screening wilh not detect all individuals with inherited hyperlipiademias, but only those from families wheres early CHD or lipid problem is known.

For screening, a blood total cholesterol ando triglyceride are performed; if these are definitely oto possibly abnormal (cholesterol $>5.2 \mathrm{mmol} / 1$; tri glycerides $>1.5 \mathrm{mmol} / \mathrm{l})$, they should be repeated $\stackrel{?}{+}$ together with estimates of high and low densityo lipoproteins (HDL and LDL), and possibly also? lipoprotein (a) (see below). As there is no routines 
diagnostic test available for either $\mathrm{FH}$ or $\mathrm{FCH}$, diagnosis is made on the basis of blood lipid and lipoprotein concentrations in the patient and family.

Familial hypercholesterolaemia is dominantly inherited, predisposes to premature CHD and can be diagnosed in affected invididuals at any age after the first year by measurement of blood lipids and lipoproteins, and by the demonstration of similar lipid abnormalities in first degree relatives. When FH is detected in childhood, diagnosis predates the development of significant coronary atherosclerosis. Blood cholesterol and LDL can be lowered by diet or drug treatment, ${ }^{2}$ and studies in adults have shown that this delays or prevents the onset of coronary heart disease. The essentials for a screening programme are thus met; however, to be effective in delaying the onset of CHD, treatment has to be prolonged, and long-term compliance with both diet and drugs is only moderate, even where there is a strong family history of early CHD. ${ }^{3}$ The importance of avoiding smoking can be emphasized, and if this advice is heeded there is a lowering of the CHD risk even if blood lipids are not significantly lowered.

Familial combined hyperlipidaemia is dominantly inherited and affected adults have raised blood cholesterol and/or triglyceride. The blood lipid abnormalities usually do not become manifest until adulthood, although isolated hypercholesterolaemia or hypertriglyceridaemia may occur in childhood. While children should be included in family studies for suspected $\mathrm{FCH}$, normal lipids and lipoproteins in childhood do not exclude FCH and blood lipids should be remeasured in early adult life.

Raised concentrations of lipoprotein (a) ( $L p(a))$ predispose to early onset CHD, particularly when associated with raised LDL concentrations. Levels of $\mathrm{Lp}$ (a) tend to rise during childhood. Raised concentrations of $L p(a)$ have been found above the age of 10 in some children whose parents had early onset CHD. ${ }^{4}$ It may be useful to inciude $\mathrm{Lp}(\mathrm{a})$ estimation in children being screened as part of a family study, but evidence on this is lacking.

\section{Population screening}

Screening blood lipids and lipoproteins in groups of children not selected on the basis of family history of CHD is difficult to justify, except for research purposes in defining population norms. Targeted screening does not detect all individuals with inherited hyperlipidaemias, but until specific diagnostic tests are available, as well as effective and generally acceptable treatment the logistic difficulties and resource implications of population screening are enormous. In a population screen only a minority of those with raised blood cholesterol will have a familial hyperlipidaemia, and diagnosis of these will require family studies. Apart from familial hyperlipidaemias, blood cholesterol concentrations in childhood have not been shown to correlate with CHD incidence. While some degree of 'tracking' of blood cholesterol occurs with time in childhood, a single blood level in childhood is not predictive of adult levels of plasma cholesterol. Population screening will not therefore diagnose latent disease, nor is there evidence of benefit of intervention. Resources for such a programme cannot be justified. There is a case for every individual having their blood lipids measured at least once; one of the benefits would be the detection of familial conditions. Early adult life, by which time the blood lipid abnormalities of FCH are manifest, would seem the ideal time.

\section{Treating children with hyperlipidaemia}

FH is associated with increased risk of CHD developing in adult life. ${ }^{1}$ Prevention is most likely to be successful if started in early life, before the development of atherosclerosis. Reducing blood lipids and avoiding other CHD risk factors (particularly smoking) are the basis for prevention, and such treatment needs to be lifelong. A diet low in saturated fat is hypocholesterolaemic, but longterm compliance is difficult, and in many patients it is not possible to keep blood lipids within the normal range. The use of drugs requires caution in children who untreated will probably remain well for several decades. Cholestyramine and colestipol are effective in lowering blood cholesterol, and appear safe for long-term use. Fibric acid derivatives have been used in children, but their hypocholesterolaemic effect is relatively weak; statins have not yet been evaluated for safety in childhood.

\section{References}

1. Slack, J. Risks of ischaemic heart disease in hyperlipidaemic states. Lancet 1969, 2: 1380.

2. West, R.J., Fosbrooke, A.S. \& Lloyd, J.K. Treatment of children with familial hypercholesterolaemia. Postgrad Med J 1975, 51 (Suppl 8): 82-86.

3. West, R.J., Lloyd, J.K. \& Leonard, J.V. Long-term follow up of children with cholestyramine. Lancet 1980, 2: 873-875.

4. Kostner, G.M., Czinner, A., Pfeiffer, K.H. \& Bihari-Varga, M. Lipoprotein (a) concentrations as risk indicators for atherosclerosis. Arch Dis Childhood 1991, 66: 1054-1056. 


\section{Screening for hyperlipidaemia and its treatment in women and the elderly}

\section{J.P. Miller}

\section{Department of Medicine, University Hospital of South Manchester, M20 8LR, UK}

Recent intervention studies, which have demonstrated that drug treatment of hyperlipidaemia leads to a reduction in coronary events or to benefit in sequential angiographic studies, have mainly been performed in men under 65 years of age. Doubts are therefore sometimes expressed about extrapolating their conclusions to women and the elderly, a majority of whom are women.

Ultimately as many women will develop coronary heart disease (CHD) as will men, albeit about a decade later. We should not allow the relative immunity of women to CHD to distract us from the fact that it is still the major cause of morbidity and mortality in middle-aged and elderly women. It is scarely justifiable to ignore opportunities for CHD prevention in women simply because men are at higher risk. Epidemiological data suggest that conventional risk factors operate in both women and the elderly, though relative risk of coronary death is lower for hypercholesterolaemic individuals in these groups than in men aged under 65 years. The overall potential for delaying or preventing events (attributable risk) is however numerically much greater at older ages simply because clinical CHD is much commoner. The difficulties lie in selecting those individuals most likely to benefit from risk factor modification and in the absence of interventional studies to confirm the benefit suggested by the epidemiological data. The advent of more powerful lipid-lowering agents should make it possible to answer some of these questions and relevant studies are planned or under way.

The detection of all high-risk individuals requires the screening for hyperlipidaemia of all adults, say between the ages of 20 and 65 years, but many primary care teams do not have the facilities yet to undertake this, and a selective high-risk screening strategy is being adopted for both men and women. A crude rule of thumb which takes some account of the reduced risk of CHD, especially in premenopausal women, is to regard male sex as an additional risk factor when choosing subjects for selective screening. A woman with a single risk factor therefore would not automatically qualify for screening, though attention should be directed to that risk factor and she should not be exempt from standard lifestyle advice. Alternatively the same criteria can be used for selective screening of both men and women using a random non-fasting total cholesterol. Male sex (or a pre- mature menopause) can then be used as an add tional risk factor in deciding whom to recall for full fasting lipid profile and in whom to use drugs of dietary measures prove inadequate.

Where non-selective screening programmes a $\overrightarrow{\bar{t}}$ in operation over fixed age ranges the age for initiating screening in women could be delayed bf say ten years compared to that for men. Suoch measures should, however, be regarded as tempon ary to facilitate the early years of screening with the ultimate objective being to screen the whole aduft population between 20 and 65 years of age.

Systemically absorbed lipid-lowering drugs should only be given to asymptomatic women child-bearing potential if they are considered to tee at very high risk. They should be advised not conceive while on the medication. There is evidenes that at least one of the new 'statin' drugs teratogenic in animal studies. Difficulties arise when a very high risk woman has problems with subfertility and many years can pass while she 8 trying to conceive, but is not receiving treatmene The non-absorbable bile-acid sequestrating agents would seem to be a logical choice in this situation but the data sheets carry a caution about use pregnancy even for these.

After the menopause the risk of coronary disea increases markedly in women, but overall doesfnot overtake that for men. Serum cholesterol glss continues to rise until some time in the seve्gिt decade so that on average it exceeds that for ment in whom cholesterol plateaus in the fifth decade. Indeed it has been reported that as many as $30 \%$ क्f women aged 55-64 years may have serum choles? terol concentrations in excess of $7.8 \mathrm{mmol}$. Clearly it would be inappropriate for all thes women to be given lipid-lowering drugs and map agement should be predominantly dietary. HDEcholesterol concentrations are often high and the ratio of HDL to total serum cholesterol can be used together with all the usual indicators of risk making a decision about the need for drug treat ment when dietary measures have proved inade. quate. A proportion will have hypothyroidism anjel this should be adequately excluded. Consideration should also be given to the use of hormone replacement therapy (HRT), especially in those who have had a hysterectomy and could receike unopposed oestrogen, since it is with this that most of the evidence for the cardioprotective effects of HRT was obtained (see M. Seed, pp. 877-879) N

Why should non-selective screening programe mes not be extended to include those over 65 yearf The proportion of elderly patients with serum cholesterol levels exceeding abitrary cut-points necessitating individual management is large. For example, in the USA it has been estimated that -3 third of men and half of women aged 65-74 yea have cholesterol levels in excess of $6.2 \mathrm{mmo}$ 
(defined as 'high' by the National Cholesterol Education Program). It is probably not justifiable at present to institute mass screening of asymptomatic individuals in this age range until data from lipid-lowering trials are known, particularly when adequate screening programmes for younger subjects are not yet available in many areas. Lifestyle is not easy to change in the elderly but nevertheless a population strategy should at present be the principal approach for the majority.

Where an individual over 65 years has proven coronary disease, then in the author's view an active approach to detect and treat hyperlipidaemia is justified.

\section{Further reading}

Beaglehole, $\mathrm{R}$. Coronary heart disease and elderly people. $\mathrm{Br} \mathrm{Med}$ J 1991, 303: 69-70.

Castelli, W.P. Cardiovascular disease in women. Am J Obstet Gynecol 1988, 158: 1553-1556.

Denke, M.A. \& Grundy, S.M. Hypercholesterolaemia in elderly persons: revolving the treatment dilemma. Ann Intern Med 1990, 112: 780-792.

Manolio, T.A., Pearson, T.A., Wenger, N.K. et al. Cholesterol and heart disease in older persons and women: review of an NHLBI workshop. Ann Epidemiol 1992, 2: 161-176.

Miller, J.P. Significance of hyperlipidemia in the elderly. Prog Cardiol 1992, 5: 93-102.

\section{Oral contraception, hormone replacement therapy and hyperlipidaemia}

\section{Seed \\ Department of Medicine, Charing Cross Hospital, London W6 $8 R F$}

Women with severe hyperlipidaemia, such as those with familial hypercholesterolaemia $(\mathrm{FH})$, are at greatly increased risk of coronary heart disease (CHD). ${ }^{1}$ Will the use of oral contraception (OC) increase this still further? Epidemiological studies of women, both on OC and hormone replacement therapy (HRT) have not assessed their lipid status, so one in five hundred of these women will have had FH and 5-15\% depending on age are likely to have been hyperlipidaemic (cholesterol $>6.5 \mathrm{mmol} / 1$ and/or triglycerides $>1.8 \mathrm{mmol} / \mathrm{l})$ : but there has been no epidemiological study specifically to address the problem of $\mathrm{OC}$ in women with hyperlipidaemia, nor are there sufficient data from the FH register ${ }^{1}$ to assess this problem.

The effect of the administration of sex steroids on lipoprotein metabolism depends on the type, dosage, route, balance of oestrogen and progestogen both in OC and HRT, and on the underlying endocrine status of the woman. However, changes in lipoproteins which are relevant to CHD risk in both normal and hypercholesterolaemic women, are not the only metabolic effects seen in women taking sex steroids, because oral oestrogen also affects the synthesis of proteins involved in coagulation and fibrinolysis.

\section{Oral contraception}

The original epidemiological observations in women on OC in the 1960 s and early $1970 \mathrm{~s}^{2}$ showed an increased incidence of thrombosis (venous and arterial), which was related to age, weight and smoking and led to a small number of excess deaths in women on OC. More recent studies in women taking low-dose OCs (35-30 $\mu$ g ethinyloestradiol) have shown no such increase in fatal events. A study from Finland involving 1,460,000 womenyears of $O C$ use showed a relative risk of pulmonary embolus of 1.2 , but a reduced relative risk of 0.2 for myocardial infarct in the OC users. ${ }^{3}$ Another recent study of 119,000 female nurses in Boston also showed no increased death from CHD in those who had taken OC; in this study $4 \%$ were hypercholesterolaemic, $12 \%$ hypertensive and $35 \%$ smoked. ${ }^{4}$

The lipoprotein changes associated with lowdose $\mathrm{OC}$ use are small. There is a $10 \%$ reduction of HDL cholesterol by OCs containing androgenic progestogens, e.g. Norgestrel, and a $30-40 \%$ increase in serum triglyceride with the newer proges-

Table I Oral contraceptive

\begin{tabular}{llll}
\hline Name & Progestogen $(\mu g)$ & Oestrogen $(\mu g)$ & \\
\hline Mercilon & $\begin{array}{l}\text { Desogestrel } \\
150\end{array}$ & $\begin{array}{l}\text { Ethinyloestradiol } \\
20\end{array}$ & $\begin{array}{l}\text { Breakthrough } \\
\text { bleeding may be } \\
\text { a problem }\end{array}$ \\
Marvelon & Desogestrel & Ethinyloestradiol & \\
& 150 & 30 & \\
Brevinor & Norethisterone & Ethinyloestradiol & \\
& 500 & 35 & \\
Ovysmen & Norethisterone & Ethinyloestradiol & \\
& 500 & 35 & \\
\hline
\end{tabular}


togens, such as Desogestrel and Gestodene, which potentiate the effect of the oestrogen component which is also to raise serum triglyceride levels. The anti-atherogenic effect of oestrogen (reduced LDL and increased HDL) may be partly counteracted by the raised triglyceride and possible interaction between this and thrombotic factors. ${ }^{5}$ Table I indicates some OCs suitable for hyperlipidaemic subjects.

Changes in haemostatic factors are probably more important than those in lipoproteins in altering CHD risk, in both normal and hypercholesterolaemic women. Women taking low-dose oestrogen OCs have small increases, $10-15 \%$, in proteins involved in coagulation. However, because there are also concomitant increases in proteins involved in fibrinolytic activity, there may be little overall change in the thrombotic tendency of most women. Women with severe hypercholesterolaemia are at particular risk of thrombosis, because of the increased reactivity of platelets in the presence of raised LDL concentrations. In such women, progestogen only oral contraceptives (POPs) are recommended, particularly if the hyperlipidaemia is untreated.

In subjects with known hyperlipidaemia, or those with a family history of premature coronary disease or thrombosis, lipoproteins should be measured before starting OC. If lipoproteins are abnormal, they should be remeasured after 6 months of OC treatment. If there is no change from the untreated level or if the lipoproteins have an improved pattern, OC may be continued with annual follow-up. Patients with severe hyperlipidaemia which does not respond to lipidlowering therapy and who smoke, should not be prescribed the combined oral contraceptive. POPs may be used.

\section{Hormone replacement therapy}

Epidemiological studies in women taking HRT have shown that they have reduced total mortality and reduced cardiovascular mortality. ${ }^{6}$ These studies have included women who are hyperlipidaemic, who smoke and who are hypertensive.

The increase in cholesterol and LDL occurring naturally in older women can be reduced using oral oestrogen, probably as a result of restoration of LDL receptor activity. The less potent 'natural' oestrogen (so called because it is metabolized to oestradiol and oestrone) used as HRT has less effect on haemostatic factors: there is no reported increased risk of thrombosis. In women who have not undergone hysterectomy, progestogen must also be administered cyclically or at low dosage continuously to avoid endometrial cancer. The choice of progestogen is important in HRT, as the
Table II Hormone replacement therapy

\begin{tabular}{|c|c|c|}
\hline Trade name & Generic name & Dose \\
\hline Unopposed & & \\
\hline Oestrogens & & \\
\hline Progynova & Oestradiol valerate & $1-2 \mathrm{mg}$ \\
\hline Premarin & $\begin{array}{l}\text { Conjugated equine } \\
\text { oestrogen }\end{array}$ & $0.625-1.25 \mathrm{n}$ \\
\hline Estraderm 50 & Oestradiol & $\begin{array}{r}25,50-100 \text { व } \\
\mu \mathrm{g} / 24 \text { hours }\end{array}$ \\
\hline
\end{tabular}

Any of the above, together with progestogen cyclicall 5 titrated to achieve a minimal bleed.

Progestogens

Provera

Medroxyprogesterone $2.5-5 \mathrm{mg}$ acetate

Micronor Norethisterone acetate $350-700 \mu \mathrm{g}$

Duphaston

Dydrogesterone

$10 \mathrm{mg}$

effect of progestogens on lipoproteins is to oppose the favourable effect of oestrogen: this appears to relate to their androgenicity. Thus, the reduction in LDL and increase in HDL with oestradiol is lessened with the addition of an androgenic proges togen, such as Norgestrel. Two progestogens with less androgenic activity are medroxyprogesterơneo acetate and dydrogesterone. Both should be $\mathrm{gt} t+0$ rated to give a minimum bleed if taken cyclicallylow dose of norethisterone acetate 350-700 $\mu$ g (Micronor one to two tablets daily) will also protece the endometrium. In women who have undergone hysterectomy, unopposed oestrogen can be used $\bar{Q}$ (Table II)

Experimental work on monkeys has shown reduced stiffness of atheromatous coronary arteries in oestrogen takers, ${ }^{7}$ and oestrogen may also reduce oxidation of LDL. Oestrogen use in secon:dary prevention of coronary disease may be effec을 tive. ${ }^{8}$ In the meantime, in patients with $\mathrm{FH}$, who donot have significant CHD (an exercise electrocar 3 . diogram may be helpful to clarify this), provided they are non-smokers, HRT is recommended together with low-dose aspirin. At present $\infty$ cautious approach is advocated in those who do have CHD.

\section{Conclusions}

Women with undiagnosed hyperlipidaemia have already taken part in epidemiological studies of $\mathrm{OC}$ and HRT. These studies have not demonstrated any increase in thrombosis, rather a decrease in mortality and myocardial infarct. The changes in lipoproteins on OC are very small. Those on HRTOे are theoretically antiatherogenic. The beneficia $\mathbb{D}^{+}$ 
action of oestrogen on the arterial wall may also be important. Changes in proteins involved in clotting urge caution with combined oral contraception in patients with severe hypercholesterolaemia or hypertriglyceridaemia, particularly if they are smokers, and not treated with lipid-lowering therapy.

General practitioners and family planning clinics at present take a history from patients wishing to start the oral contraceptive. If the medical history includes thrombosis, the pill is contraindicated. If there is a family history of premature cardiovascular disease, or if the patient has any physical signs of hyperlipidaemia, lipids should be measured. Blood pressure is routinely checked on women starting the OC, opportunistic measurement of lipids could be also carried out. In the small number of women in this young age group likely to be hyperlipidaemic, treatment of the hyperlipidaemia by lifestyle advice sometimes combined with drug therapy, should be instituted.

\section{References}

1. Scientific Steering Committee on behalf of the Simon Broom Research Group. Risk of fatal coronary heart disease in familial hypercholesterolaemia. Br Med J 1991, 303: 893-896.

2. Mann, J.I., Vessey, M.P., Thorogood, M. \& Doll, R. Myocardial infarction in young women with special reference to oral contraceptive practice. $\mathrm{Br}$ Med J 1975, 2: 241-245.

3. Hirvonen, E. Fatal cardiovascular complications during the use of low-estrogen oral contraceptives compared with IUD use and live births associated with deaths in a ten year period 1975-1984 in Finland. Adv Contracept 1988, 4(1): 62-68.

4. Stampler, M.J., Willett, W.C., Colditz, G.A., Speizer, F.E. \& Hennekens, C.H. A prospective study of past use of oral contraceptive agents and risk of cardiovascular disease. $N$ Engl $J$ Med 1988, 319: 131-137.

5. Skouby, S.O. \& Jersperson, J. Oral contraceptives in the nineties - facts and fiction. First European conference on sex steroid and metabolism. Am J Obstet Gynecol 1990, 163/1 (ii).

6. Bush, T.L. The epidemiology of cardiovascular disease in post menopausal women. Ann N Y Acad Sci 1990, 592: 263-271.

7. Adams, M.R., Clarkson, T.B., Shiveley, C.A., Parks, J.S. \& Kaplan, J.R. Oral contraceptives, lipoproteins and atherosclerosis. Am J Obstet Gynecol 1990, 163: 1388-1393.

8. Sullivan, J.M., Zwaag, R.V., Hughes, J.P., Maddock, V. \& Kroetz, F.W. Estrogen replacement and coronary disease. Arch Int Med 1990, 150: 2557-2562.

\section{Fish oil and heart disease}

\section{T.A.B. Sanders}

\section{Department of Nutrition \& Dietetics, King's College London, Campden Hill Road, London W8 $7 A H, U K$}

Populations that eat large amounts of oily fish or marine mammals have low rates of coronary heart disease (CHD). Prospective and case control studies also find that oily fish consumption in Western populations is associated with a decreased risk of coronary disease independent of other major risk factors such as smoking, high blood pressure and serum cholesterol levels.

Recent evidence suggests that oily fish consumption is of benefit following a heart attack. Approximately 2,000 men who had recently recovered from a myocardial infarction were randomly allocated to different dietary treatments. ${ }^{1}$ Half the subjects received dietary advice to consume two portions of oily fish a week (sardines, pilchards, mackerel, salmon, herring). A factorial design was used to test three dietary factors independently fat, fish and fibre. Not all of the subjects given the fish advice were able to tolerate the fish and were instead given fish oil capsules. At six months about $14 \%$ of the fish advice group were taking capsules. No benefit was seen from decreasing fat or increasing fibre intake, but the group who were given advice to eat oily fish showed a significant $(29 \%)$ reduction in risk of death over the subsequent 2 years.

Oily fish contains several nutrients - retinol, vitamin $D$, taurine, iodine, selenium and n-3 fatty acids (that are scarce in other foods), consequently small intakes of fish can make a substantial contribution to dietary intake. Most attention has focused on potential health benefits of the n-3 fatty acids, in particular eicosapentaenoic acid (20:5n-3: EPA) and docosahexaenoic acid (22:6n-3: DHA). Attention focused on these fatty acids because they were present in high concentrations in the plasma lipoproteins of Eskimos. These fatty acids are derived almost exclusively from the marine food chain and have been shown to exert a number of physiological effects that are of relevance to CHD.

Neither EPA nor DHA can be synthesized de novo but they can be synthesized to a limited extent from $\alpha$-linolenic acid, which is found in certain vegetable oils. DHA is believed to be involved in the transmission of electrical signals in both the retina and brain, EPA is believed to play an important role in modulating the production of active eicosanoids (prostaglandins, leukotrienes and thromboxanes) derived from arachidonic acid. The consumption of fish oil containing EPA decreases the proportion of arachidonic acid in membrane lipids and replaces it with EPA. EPA can be converted to eicosanoids but they tend to be less active than those derived from arachidonic acid. Consequently the effect of replacing arachidonic acid with EPA is to decrease the production of prothrombotic eicosanoids. ${ }^{2}$

A number of trials have been carried out with fish oil supplements such as MaxEPA. Ten grams of MaxEPA provided $1.8 \mathrm{~g}$ EPA and $1.2 \mathrm{~g} \mathrm{DHA.} \mathrm{A}$ consistent observation has been that moderate intakes of oily fish (in excess of $100 \mathrm{~g} /$ day) or fish oil 
supplements $(10-20 \mathrm{ml} /$ day) lead to a prolongation of template bleeding time. Fish oil treatment prolongs bleeding time when administered with aspirin to a greater extent than with aspirin alone. However, there is no evidence that post-operative blood loss is increased following fish oil administration. No consistent changes in clotting factors or fibrinolytic activity have been noted in subjects given fish oil supplements. Fish oil is only a weak inhibitor of platelet aggregation compared with aspirin, but is a potent inhibitor of platelet adhesion. ${ }^{3}$ This latter effect may explain the prolongation of bleeding time and could explain its protective effect against CHD.

The majority of studies on the effects of fish oil supplements on blood pressure have observed modest reduction in both normotensive and mildly hypertensive individuals. Overall the studies indicate a small blood pressure lowering effect with EPA and DHA intakes in excess of $3 \mathrm{~g}$ a day. The effect appears to be more apparent in subjects with mild to moderate hypertension than normotensive individuals and appears to persist as long as the intake levels are maintained. ${ }^{4}$ In general, however, reductions of more than $3-5 \mathrm{mmHg}$ in systolic and diastolic pressure cannot be expected.

Feeding of fish oil to experimental animals leads to marked changes in the composition of cardiac membrane phospholipid, in particular the proportion of DHA is increased and that of arachidonic acid is decreased. Fish oil supplementation in experimental animals has the effect of decreasing the damage caused following experimental myocardial infarction. Other studies have also shown that susceptibility to cardiac arrhythmias is decreased in both rats and primates fed diets containing fish oil. However, the amounts of fish oil used in these studies have been relatively high, therefore extrapolation to human studies using lower amounts of fish oil may not be relevant.

Does fish oil lower plasma cholesterol? The answer to this question is generally no. Fish oil, however, has a marked plasma triglyceride lowering effect. ${ }^{5,6}$ This is due to an effect on the secretion and synthesis of triglycerides in very low density (VLDL) and intermediate density lipoprotein (IDL). In patients with Type III $^{7}$ and Type V hyperlipoproteinaemia, where total cholesterol concentrations are elevated due to large increases in the number of IDL and VLDL particles, fish oil treatment usually leads to a reduction in total cholesterol. Fish oil would not be expected to reduce plasma triglycerides in patients with lipoprotein lipase deficiency.

As a rule low density lipoprotein (LDL) levels are either unaffected by fish oil treatment in the doses commonly used $(5-15 \mathrm{ml} /$ day $)$, or may be slightly elevated, particularly in patients with the Type IV phenotype. A similar phenomenon is observed with other triglyceride-lowering agents气 such as fibrates. Increases in LDL cholesterol havez been observed in patients with insulin-dependento diabetes mellitus as well as in those with non- - . insulin-dependent diabetes mellitus treated with $\vec{F}$ fish oil. Glucose control may also be impaired in non-insulin-dependent diabetes mellitus. Increases in high density lipoprotein (HDL) and $\mathrm{HDL}_{2} \overline{\bar{c}}$ cholesterol concentrations have been observed in $\vec{\sigma}_{\bar{\sigma}}$ several studies where subjects have been given $\cong$ 2-5 $\mathrm{g}$ of EPA and DHA a day and this is accompanied by a decrease in the activity of cholesterol $\vec{O}$ transfer protein. Very high intakes of fish oil (in excess of $40 \mathrm{ml} /$ day), on the other hand, decrease $\vec{\omega}$ HDL cholesterol levels. Claims that fish oil supple-aे ments lower $L p(a)$ levels in individuals with high levels have not been confirmed.

It is questionable whether the changes in plasma@ lipids brought about by fish oil have a beneficial ${ }^{\infty}$ influence with regard to risk of atherosclerosis. On Reductions in VLDL, IDL and chylomicron remnants are achieved, but LDL cholesterol levels mayo be increased. The consumption of fish oil changes the composition of lipoprotein particles and this $\vec{Z}$ may affect their subsequent metabolism. EPA and DHA are particularly prone to peroxidation and $\stackrel{\Phi}{3}$ the consumption of fish oil leads to an increasedo requirement for antioxidant vitamins. This may be of significance as oxidatively modified low densi⿺辶 $\overrightarrow{0}$ lipoprotein is believed to be necessary for foam cell 10 formulation, which is one of the first stages in the formation of an atherosclerotic plaque. In theory? this would suggest that fish oil may promote atherosclerosis; however, fish oils rich in EPA and DHA have been found to inhibit development of experimental atherosclerosis in pigs, dogs and $\stackrel{\varrho}{\Rightarrow}$ monkeys, even in the presence of a high plasma cholesterol concentration. This implies that protec- 3 tion is afforded by a mechanism independent of plasma lipid concentration. Several studies have noticed that fish oil inhibits cellular proliferation $\frac{0}{3}$ and this may be due to effects of EPA and DHA on the production of cytokines and factors that stimulate cellular proliferation.

Several trials have examined the effects of fish oil $\frac{2}{3}$ on restenosis following coronary angioplasty. 0 Although early studies showed some benefits from supplementation, others show no benefit. Overall there is a non-significant trend for the restenosis rate to be about $20 \%$ lower with fish oil treatment. $N$ Unfortunately most of the studies compared the $N$ incidence of fish oil in addition to aspirin treatment $N$ and consequently any prostaglandin-mediated ${ }^{\omega}$ effects that might be exerted by fish oil may well be masked by aspirin. 


\section{Conclusion}

Fish oil exerts a number of physiological effects that may be relevant to coronary heart disease. However, fish oil is not useful for lowering plasma cholesterol concentration. Most studies have used relatively high intakes of EPA and DHA, usually in excess of $2-3 \mathrm{~g}$ a day. That is equivalent to an amount provided by $100-200 \mathrm{~g}$ portion of oily fish. To date the only accepted clinical indication for the use of fish oil has been in the management of hypertriglyceridaemia. The full potential of these compounds is still under investigation. In the meantime it makes sense to encourage the consumption of fish as an alternative to food high in saturated fatty acids. This would be in accord with current dietary guidelines.

\section{References}

1. Burr, M.L., Fehily, A.M., Gilbert, J.F. et al. Effects of change in fat, fish and fibre intakes on death and myocardial reinfarction: Death and Reinfarction Trial (DART). Lancet 1989, 2: 757-760.

2. Leaf, A. \& Weber, P.C. Cardiovascular effects of n-3 fatty acids. $N$ Engl J Med 1988, 318: 549-557.

3. Lei, X.L. \& Steiner, M. Dose response of dietary fish oil supplementations on platelet adhesion. Arterioscler Thromb 1991, 11: 39-46.

4. Bønaa, K.H., Bjerve, K.S., Straume, B., Gram, I.T. \& Thelle, D. Effect of eicosapentaenoic and docosahexaenoic acids on blood pressure in hypertension. A population-based intervention trial from the Tromsø study. N Engl J Med 1990, 322: 795-801.

5. Sanders, T.A.B. Influence of n-3 fatty acids on blood lipids. World Rev Nutr Diet 1991, 66: 358-366.

6. Harris, W.S. Fish oils and plasma lipids in lipoprotein metabolism in humans: a critical review. J Lipid Res 1989, 30: 785-787.

7. Mölgaard, J., von Schenck, H., Lassvik, C., Kuusi, T. \& Olsson, A.G. Effect of fish oil treatment on plasma lipoproteins in type III hyperlipoproteinaemia. Atherosclerosis 1990 , 81: $1-9$.

\section{'Lipid-friendly' antihypertensives}

\section{D.J. Betteridge}

\section{Department of Medicine, University College and Middlesex School of Medicine, 5th Floor, Jules Thorn Institute, Middlesex Hospital, Mortimer Street, London, WIN 8AA, UK}

There is general agreement that the major risk factors for coronary heart disease (CHD) are high blood cholesterol levels, elevated blood pressure and cigarette smoking. Furthermore, when these risk factors are present in the same individual CHD risk multiplies.

Blood pressure shows a gradient of risk for CHD mortality similar to that observed for serum cholesterol. However, whereas there is convincing evidence of the benefit of pharmacological reduction of blood cholesterol in terms of CHD, the evidence of benefit from pharmacological modification of blood pressure is less clear. There is no doubt that antihypertensive therapy helps protect patients from stroke, renal failure and congestive heart failure, but antihypertensive therapy has not been shown convincingly to protect against CHD. A recent meta-analysis of primary prevention trials involving antihypertensive therapy has indicated a reduction in CHD, but less than would be predicted from the strength of the epidemiological association. ${ }^{1}$ A possible explanation for this discrepancy involves the adverse effects of some antihypertensive agents on lipid and lipoprotein metabolism. Intervention trials initiated in the 1960s and 1970s involved thiazide diuretics and beta-adrenergic blocking agents which have subsequently been found to affect serum lipid and lipoprotein concentrations adversely. ${ }^{2,3}$

Those beta-adrenergic blocking drugs which lack alpha-blocking effects, intrinsic sympathetic activity or vasodilator properties, tend to increase serum triglyceride levels $(20-50 \%)$ and to reduce HDL cholesterol levels $(-15 \%)$. Selective betablockers have similar but less pronounced effects to non-selective agents.

Thiazide diuretics produce increases in serum triglycerides by up to $50 \%$ with more modest effects on serum cholesterol (10-20\%). HDL cholesterol concentrations are unaffected.

The calcium antagonists and angiotensin-converting enzyme inhibitors appear to have neutral effects on serum lipids, whilst the selective alpha $a_{1}$ adrenergic blocking agents may have small 'favourable' effects on cholesterol and triglyceride levels. $^{4}$

The potential adverse effects of some antihypertensive agents should be considered when planning therapy. There is no doubt that adverse lipid profiles commonly occur in hypertensive subjects. ${ }^{5}$ This clustering of risk factors may in a proportion of hypertensive peoples be related to the syndrome of resistance to insulin-mediated glucose uptake. ${ }^{6}$

Fasting cholesterol, triglyceride and HDL cholesterol measurements should be part of the management of all hypertensives. Lifestyle measures (weight reduction, modified fat diet) should be instigated where lipid and lipoprotein levels are outside the guidelines for optimal levels. A hypertensive agent without adverse metabolic effects should be used if possible. This is not to say that where necessary beta-blockers may not be used in angina or postmyocardial infarction.

Hypertension is common in the diabetic population particularly in those who are non-insulindependent and in insulin-dependent patients with proteinuria. Both these groups of patients tend to have abnormalities of serum lipid and lipoprotein 
concentrations. Again, where appropriate, metabolically neutral antihypertensive agents should be chosen. The thiazide diuretics and beta-adrenergic blocking agents have other potential adverse effects in diabetes: thiazides adversely affect glucose tolerance and beta-blockers may mask symptoms and delay recovery from hypoglycaemia. ${ }^{7}$

\section{References}

1. Collins, R., Peto, R. \& MacMahon, S. Blood pressure, stroke and coronary heart disease. Part 2 . Short term reductions in blood pressure: overview of randomised drug trials in their epidemiological context. Lancet 1990, 335: 827-838.

2. Weidman, P., Uehlinger, D.E. \& Gerber, A. Antihypertensive treatment and serum lipoproteins. $J$ Hypertens 1985, 3: 297-306.

3. Krone, W. \& Nagele, H. Effects of antihypertensives on plasma lipid and lipoprotein metabolism. Am Heart J 1988, 116: 1729-1734.

4. Krone, W. \& Muller-Wieland, D. Hyperlipidaemia and hypertension. Bailliere's Clin Endocrinol Metab 1990, 4: 833-850.

5. MacMahon, S.W. \& MacDonald, C.-J. Antihypertensive treatment and plasma lipoprotein levels the association in data from a population study. Am J Med 1986, 80 (Suppl. 2A): $40-47$.

6. Reaven, C.-M. Role of insulin resistance in human disease. Diabetes 1988, 37: 1595-1606.

7. Stein, P.P. \& Black, H.R. Drug treatment of hypertension in patients with diabetes mellitus. Diabetes Care 1991, 14: 425-448.

\section{Is intervention cost effective?}

J.P.D. Reckless

Royal United Hospital, Bath BA1 3NG, UK

\section{Introduction}

National resources are insufficient to meet all potential demands, so economic considerations need to play a part in rational decisions for allocation of medical care, such as for coronary heart disease (CHD). Various measures of economic performance are available. Cost effectiveness looks at cost per unit of outcome. This can be extended as cost-utility analysis to measure quality as well as survival, years of life saved being adjusted for quality in the quality adjusted life year or $Q A L Y$.

\section{Cost-utility considerations for hyperlipidaemia}

History, symptoms and signs may detect some individuals with significant hypercholesterolaemia, but a substantial proportion will not be identified unless screened. ${ }^{1}$ All the population may be screened, but not all will require treatment. Cholesterol is

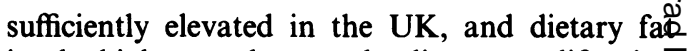
intake high enough, to make dietary modificatio appropriate for most people. Dietary change with out cholesterol measurement involves advice tethose who may not benefit, removal of potentia毒 motivation from knowing a cholesterol level, and failure to detect high-risk individuals and genetig hyperlipidaemias.

It is essential that drug treatment is targeted $t \frac{\phi^{2}}{2}$ people at high CHD risk, because of potential drug side effects, effort involved, and overall cost te individual and/or community of long-term medica $\vec{O}$ tion. A fixed cholesterol concentration at which to start drug treatment is not appropriate. One should consider an individual's potential risk that theio level of cholesterol poses in the light of their overa risk factor status. Treating individuals with exist: ing CHD, with multiple risk factors, or with single gene lipid disorders will maximize benefit, limis drug usage and overall cost, and give best economi $\xi_{0}$ performances. Such careful choice does require $\mathbb{P}$ degree of understanding and training of doctors im the inter-relationships of multiple risk factors, and does require intervention on all, and not just on one, modifiable risk factors.

In the UK, cost-utility estimates ${ }^{2,3}$ have bee made for opportunistic screening for adults of working age routinely visiting the primary c\&re team. Non-attenders include higher risk indiuedes uals, but it is more cost effective to approach the not screened at the end of a screening programen than attempt mass screening. In a ten year proß gramme $^{2}$ overall cost per QALY for those aged 20-65 years was $£ 550$. Costs per QALY vary bo gender and age reflecting differences in CHD risk and were $£ 7,980$ and $£ 280$ for those aged $20-39$ an 40-65 years, respectively, and for males anक females aged $20-65$ were $£ 370$ and $£ 1,090$, respec $s$ tively. Another set of calculations ${ }^{3}$ for a life-lon programme gave a QALY cost of $£ 2,979$ for age 40-69 years, being $£ 1,957$ for males and $£ 6,521$ fo females. These figures assume a 4-5\% drug usagerate, and the $£ 2,979$ QALY cost falls to $£ 1,481$ an $\bar{\Phi}$. $£ 831$ at half and quarter drug usage rates, respec tively. Drug usage in the UK is now only abou@ $0.01-0.02 \%$. Expenses for screening, counselling and dietary management are $<25 \%$ of total costs and without drug costs the QALY costs have beer calculated as $£ 120^{2}$ or $£ 176 .{ }^{3}$ Critical consideration. must therefore be given to drug choice and effectiveness, drug price, and particularly to the choice of patients treated.

Presence of clinical CHD increases cost effective ${ }_{\sigma}^{\omega}$ ness as the risk of further CHD events is high. Thus the costs per QALY (including drug therapy) are $£ 223$ and $£ 2,598$ for males aged $40-69$ years witkp and without CHD, respectively. ${ }^{3}$ It is more cosito effective to intervene in those with multiple CHD risk factors; for example, hypertensive male् smokers of 40-69 years have a QALY cost of $£ 71$ 20 
compared to $£ 4,076$ for normotensive nonsmokers. ${ }^{3}$ For comparison, the QALY cost for advice on smoking cessation is under $£ 100$. Most studies consider single factor intervention, but in clinical practice, and for best cost-benefit, intervention on all modifiable factors is necessary. On purely economic grounds it may be cheaper to delay risk factor treatment until the fifth decade, on the basis, for example, that two thirds of benefit of effective cholesterol lowering may be evident within 2-3 years of intervention. To a lesser extent, cost may rise somewhat per year of treatment in the seventh and eighth decades of age, as the predictive power of cholesterol is reduced with competing causes of mortality.

Wide cost estimates have been made for cholesterol intervention. For cholestyramine, Oster and Epstein ${ }^{4}$ calculated the cost per life year saved as $£ 30-£ 60,000$. An analysis from West Germany ${ }^{5}$ has produced a total cost per life year saved of $£ 10,000$ for men aged under 60 years, but in setting a goal of LDL-cholesterol of $<4.15 \mathrm{mmol} / 1$ is greatly influenced by drug use in $6.2 \%$ of people. In contrast, individual spontaneous implementation of a dietary policy has been calculated as being a saving, and not a cost, per person per annum of $£ 140$ (late 1970s costs). ${ }^{6}$ The cost effectiveness of the HMGCoA reductase inhibitor, lovastatin, ${ }^{7}$ has been calculated for primary and secondary CHD prevention at various levels of cholesterol, with or without the presence of other risk factors. Drug treatment for primary prevention was considered more cost effective for men than women, at higher than lower cholesterol levels, and much more so when multiple risk factors were present. For secondary prevention, cost effectiveness ratios were better still, and treatment of younger men and women with CHD showed savings rather than costs, therapy therefore saving both lives and money. ${ }^{7}$

Estimates of cholesterol lowering have previously been based on major double-blind placebo-controlled studies, but drug usage in clinical practice is adjusted to individual patient need and response. Estimates of costs have considered potential reduction in total (or LDL) cholesterol, but not changes in triglycerides or HDL cholesterol, and this is also true for drug effects. Recent drugs are more potent, easier to take, and the degree of cholesterol lowering is easier to predict. With increasing use, and patent changes with time, relative drug costs can be expected to fall. All these changes may improve cost-utility comparisons.

Marked changes in costs can occur with apparently modest changes in the necessary assumptions. Thus the QALY costs should be looked upon as an approximate and not a precise instrument. They do allow sensitivity analyses, to help give economic support to clinical treatment guidelines, and they do form a basis on which cost-utility ratios can be compared for prevention versus treatment of CHD, or between prevention programmes against different diseases. For coronary artery bypass grafting for main vessel disease, and for heart transplantation, QALY costs have been calculated as $£ 1,300$ and $£ 6,200$, while breast cancer and cervical cancer screening programmes have both been costed at about $£ 5,500$.

\section{Summary}

1. Opportunistic screening by the primary health care team is more cost effective than mass screening.

2. Ideally all adults should have a cholesterol measurement.

3. Dietary counselling should be offered, following European Atherosclerosis Society guidelines.

4. Drug treatment should be preceded by multiple lipid measurements and full dietary management.

5. Where logistic or other considerations limit initial cholesterol screening programmes, preference should be given to individuals with known CHD, those with a family history of premature CHD or of hyperlipidaemia, those with other known CHD risk factors, to older rather than younger persons, and to males before females.

6. Screening should be for all risk factors.

7. Intervention should not be on one, but on all modifiable risk factors.

8. Drug usage for hypercholesterolaemia should follow consideration of an individual's overall CHD risk and not just a single factor. Careful drug choice is cost effective.

\section{References}

1. Mann, J.I., Lewis, B., Shepherd, J. et al. Blood lipid concentrations and other cardiovascular risk factors: distribution, prevalence and detection in Britain. $\mathrm{Br}$ Med $J$ 1988, 296: 1702-1706.

2. Standing Medical Advisory Committee. Blood cholesterol testing and the cost-effectiveness of opportunistic cholesterol testing. Report to the Secretary of State for Health, Department of Health, London, 1990.

3. Reckless, J.P.D. Cost-effectiveness of clinical care for hyperlipidaemia. In: Lewis, B. \& Assmann, G. (eds) The Social and Economic Contexts of Coronary Prevention. Current Medical Literature, London, 1990, pp. 94-103.

4. Oster, G. \& Epstein, A.M. Cost-effectiveness of antihyperlipemic therapy in the prevention of coronary heart disease. The case for cholestyramine. JAMA 1987, 253: 2381-2387.

5. Assmann, G. \& Schulte, H. Primary prevention of coronary heart disease in the Federal Republic of Germany: Analysis of cost-effectiveness. Drugs 1990, 40 (Suppl 1): 33-37.

6. Berwick, D.M., Cretin, S. \& Keeler, E.B. Cholesterol, Children and Heart Disease: An Analysis of Alternatives. Oxford University Press, New York, 1980.

7. Goldman, L., Weinstein, M.C., Goldman, P.A. \& Williams, L.W. Cost-effectiveness of HMG-CoA reductase inhibition for primary and secondary prevention of coronary heart disease. JAMA 1991, 265: 1145-1151.

8. Reckless, J.P.D. The economics of cholesterol lowering. Bailliere's Clin Endocrinol Metabol 1990, 4: 947-972. 